\title{
Comment on "Aerobic exercise effects in renal function and quality of life of patients with advanced chronic kidney disease"
}

\author{
Fangfang Li \\ (iD) Rong $\mathrm{Yu}^{\mathbf{1}}$ \\ (iD) Ni Han' \\ (iD) Lili Zou ${ }^{1}$
}

1. Department of blood purification Center, Yantai Yuhuangding Hospital, Qingdao University Medical College, Yantai, Shandong, 264000, China.

Dear Editor,

We read with great interest the study by Calvo-Lobo et al. ${ }^{1}$ in which they demonstrated that aerobic exercise may cause improvements in renal function and in the quality of life of patients with advanced chronic kidney disease. This study further pointed out that protocols specifically for patients with advanced stages of chronic kidney disease should be carried out in order to study their effectiveness and safety. However, some concerns should be addressed.

To begin with, control subjects were not recruited in this study. In my view, there should be two groups, one group to complete the exercises and another group that is exercise-free. Additionally, the small sample is also a limitation of this study.
The description of statistical analysis should also be revised. In the results section, the author described that "the other renal function parameters did not show any statistically significant differences". The sentence should be revised as "no statistically significant was found for the other renal function parameters". Because "statistically significant difference" and "no statistically significant was found for difference" have different meanings.

\section{REFERENCES}

1. Calvo-Lobo C, Neyra-Bohorquez PP, Seco-Calvo J. Aerobic exercise effects in renal function and quality of life of patients with advanced chronic kidney disease. Rev Assoc Med Bras. 2019;65(5):657-62 